\title{
Protein Molecular Dynamics With Electrostatic Force Entirely Determined by a Single Poisson-Boltzmann Calculation
}

\author{
Ben Zhuo Lu, ${ }^{1,2}$ Wei Zu Chen, ${ }^{2}$ Cun Xin Wang, ${ }^{1,2 *}$ and Xiao-jie Xu ${ }^{3}$ \\ ${ }^{1}$ Department of Astronomy and Applied Physics, University of Science and Technology of China, Hefei, China \\ ${ }^{2}$ Center for Biomedical Engineering, Beijing Polytechnic University, Beijing, China \\ ${ }^{3}$ College of Chemistry and Molecular Engineering, Peking University, Beijing, China
}

\begin{abstract}
The electrostatic force including the intramolecular Coulombic interactions and the electrostatic contribution of solvation effect were entirely calculated by using the finite difference Poisson-Boltzmann method (FDPB), which was incorporated into the GROMOS96 force field to complete a new finite difference stochastic dynamics procedure (FDSD). Simulations were performed on an insulin dimer. Different relative dielectric constants were successively assigned to the protein interior; a value of 17 was selected as optimal for our system. The simulation data were analyzed and compared with those obtained from 500-ps molecular dynamics (MD) simulation with explicit water and a 500-ps conventional stochastic dynamics (SD) simulation without the mean solvent force. The results indicate that the FDSD method with GROMOS96 force field is suitable to study the dynamics and structure of proteins in solution if used with the optimal protein dielectric constant. Proteins 2002; 48:497-504. $\odot 2002$ Wiley-Liss, Inc.
\end{abstract}

Key words: protein dielectric constant; solvent effect; finite difference stochastic dynamics; insulin dimer

\section{INTRODUCTION}

The importance of solvent effects in determining solute properties has been known for many years. To treat the solvent effect theoretically, an explicit model including both the solvent and solute molecules is widely used in molecular modeling, ${ }^{1-3}$ in which nonbonded van der Waals and electrostatic terms account for the intermolecular interaction in detail. A proper description at the molecular level of solvent effects requires the calculation of the mutual interactions of a large number of molecules and the averaging of these over many solvent configurations. The daunting computational requirements of this approach have been partially overcome through theoretical advances and by continuing enhancements in computational power. Nevertheless, for many applications, such explicit treatment of solvent molecules and mobile ions is not feasible.

An alternative approach is to treat the solvent with a continuum model, in which a potential of mean force of solvent is introduced to describe the average solvent effect.
The mean effect of solvation has two components: the electrostatic term and the non-polar part (including solutesolvent van der Waals interactions and the hydrophobic effect). It is common practice to assume that the latter contribution is related to the solvent-accessible surface area (ASA) of the solute. ${ }^{4,5}$ However, these semi-empirical treatments are generally limited to calculations of solvation energy or free energy. It is of technically difficult and CPU-consuming to directly calculate the solvation force resulting from a variation of ASA. As for the electrostatic part of the solvation effect, it can be determined in detail by solving the Poisson-Boltzmann equation (PBE). Many efforts have been made to incorporate the solvent effect into molecular mechanics or MD simulation using the PBE. ${ }^{6-11}$ Two different numerical strategies are often used to solve the PBE: the boundary elementary method (BEM) ${ }^{6,10,11}$ and the finite difference Poisson-Boltzmann method (FDPB). ${ }^{7-9,12}$ Problems with obtaining a fast and proper triangulation of the molecular surface have been recognized as an obstacle to use of the BEM with macromolecule with "pinched" surface regions that are often found in deep crevices and internal cavities. The FDPB method has been employed successfully in numerous studies of proteins. ${ }^{13-18}$ Progress has been made in attempts to directly incorporate the $\mathrm{PB}$ equations into molecular force fields in dynamical studies in a number of cases. ${ }^{8,9,19-21} \mathrm{In}$ the usual FDPB method, the entire electrostatic force is divided into two parts, one the intramolecular Coulombic interaction, and the other related to the solvation force. The second term is determined by the FDPB method and is added to the intramolecular force that is a direct Coulomb interaction computed with the conventional molecular mechanics force field..$^{7,9,21}$ Most previous FDPB dynamics simulations were performed on small molecules, and only a few protein structures were usually studied using this

Grant sponsor: Chinese Natural Science Foundation; Grant numbers: 29992590-2, 30170230, 10174005; Grant sponsor: Beijing Natural Science Foundation; Grant number: 5992002; Grant sponsor: Science and Technology Development Project of Beijing City Education Committee.

*Correspondence to: Prof. Cun Xin Wang, Center for Biomedical Engineering, Beijing Polytechnic University, Beijing 100022, China. E-mail: cxwang@bjpu.edu.cn

Received 8 November 2001; Accepted 2 April 2002 
method. ${ }^{18}$ Recently, David et al. performed simulations on HIV protease, in which they used the UHBD program with the FD Poisson equation method with the CHARMM22 parameters and compared the simulation results with those obtained with a generalized Born solvent model. ${ }^{17}$ The electrostatic energy and atomic forces were also determined by the FDPB procedure, which can save much computational time in the calculation of the electrostatic interactions.

One of our goals is to develop the FDPB procedure ${ }^{22}$ to combine with SD simulations ${ }^{23}$ (FDSD) in order to perform simulations on proteins. In this FDSD method, the entire electrostatic interaction (including the intramolecular Coulombic interactions and the electrostatic solvation force) is determined by the PB solution, instead of only the electrostatic solvation force. This work focuses on the implementation of dynamics simulation, and the force calculation is preferred to the energy or free energy calculation. Therefore, because of the above-mentioned difficulties in incorporating the non-polar solvation force on solute atoms into dynamics simulation, this part of the solvation effect is not taken into account in our protocol.

As Schutz and Warshel ${ }^{24}$ pointed out, the value of the dielectric constant of a protein is not universal; it is entirely dependent on the way used to define this constant and on the model used. As suggested in the literature, ${ }^{24}$ a large dielectric value $(>10)$ may be proper for chargecharge interactions. In fact, we failed when a small dielectric constant (especially equal to 1) was used in our FDSD simulation under different precision of grid mesh. Therefore, a dielectric value must be selected properly for the simulation system interior. In order to determine an optimal value, we have performed a series of simulations with different values of the dielectric constant, for some of which different grid mesh were also used to investigate their influence on the accuracy of simulation results. Our criterion for what is its proper value is based on the requirement that the conformation should be maintained, but it may not guarantee the correct electrostatic energies in protein, which vary when different dielectric values are used. In our work, in which we have used the insulin dimer, we find that the most commonly suggested values of 1,2 , or 4 for the interior dielectric constant $t^{9,12,13,17,21}$ perform poorly. Instead, we find that a value of 17 for the interior relative dielectric constant in our system leads to good agreement with the MD simulation with explicit solvent in the dynamic and structural properties of the insulin dimer.

\section{MODEL AND COMPUTATIONAL DETAILS}

The classical treatment of electrostatic interaction in solution is based on the PBE:

$$
\nabla \cdot[\varepsilon(r) \nabla \phi(r)]-\varepsilon(r) \kappa^{2} \sinh [\phi(r)]+\frac{4 \pi \rho(r)}{k_{B} T}=0
$$

where $\phi(r)$ is the electrostatic potential, $\epsilon(r)$ is the dielectric constant, $\rho(r)$ is the charge density. The term $\kappa^{2}=$ $1 / \lambda^{2}=8 \pi e^{2} I / \epsilon k_{B} T$, where $\lambda$ is the Debye length and $k_{B}$ is the Boltzmann constant, $I$ is the ionic strength, $T$ is the absolute temperature. In our present work, different dielec- tric constants are assigned to the interior and exterior of the solute. The second term in Eq. 1 accounts for salt effects. When $\kappa=0$, Eq. 1 reduces to Poisson's equation, which in turn reduces to Coulomb's law when the dielectric constant is uniform throughout space. The details of the numerical approach utilizing relaxation iteration to FDPB algorithm can be found in Nicolls and Honig. ${ }^{25}$ The leap-frog algorithm ${ }^{26}$ is used for performing the SD integration.

Here, it is worth noting that the electrostatic force can be calculated by a direct PB method. Generally, the force can be obtained by the differential of the electrostatic potential with respect to position: $\mathrm{F}_{\mathrm{i}}=-\partial \mathrm{V} / \partial \mathrm{r}_{\mathrm{i}}, i$ denotes any of $\mathrm{x}, \mathrm{y}$, or $\mathrm{z}$ direction, $F_{i}$ is the $i$-th directional electrostatic force on any atom, $r_{i}$ is the $i$-th component of the atom's position. When the numerical solution of the PB equation is obtained, the electrostatic potential on any space point can be calculated from the resolved grid potential through interpolation method. To get the electrostatic force on an atom positioned at $\boldsymbol{r}$, i.e., the x-direction force $F_{x}$, the potential $V_{x u}$ at upstream with $\Delta x$ separation and $V_{x d}$ at downstream with $\Delta x$ separation are calculated at first, then the force $F_{x}=-\left(V_{x u}-V_{x d}\right) /(2 \Delta x)$ However, as pointed by Madura et al., ${ }^{27}$ the electrostatic free energy (and the derived forces) that result from a single PB calculation include three contributions: solvation, selfenergy of the gridded charges, and finite-difference Coulomb interaction between the gridded charges. The second term is an artifact of grid discretization, and the third term is a poor approximation at short distances to a direct Coulomb interaction between point charges. In this work, the self-energy is subtracted from the calculation of total electrostatic potential, but the force derived from this term cannot be excluded from a single PB calculation. To test the influence of the above approximation on dynamics simulation of protein, we performed the energy calculation and analyzed the results of simulations with different grid size, and also compared the results obtained from MD and SD simulations (see below).

However, the FDPB interaction also includes Coulombic interactions between atoms directly connected by chemical bonds (first and second neighbors, and in some cases third neighbor) as in Schaefer et al. ${ }^{15}$ This is inconsistent with the requirement of the GROMOS96 force field in the MD simulation because these atoms are excluded from the summation of the electrostatic interaction. Therefore, for each charged atom, the Coulombic interactions on which imposed by its first, second and in some cases third neighbor atoms should be subtracted from that calculated from the FDPB method.

The crystal structure of Pig Insulin Dimer was taken from the Protein Databank ${ }^{28}$ containing 102 amino acid residues in total. We have selected insulin dimer as a test system of moderate size with an experimental structure determined at high resolution. The dimer consists of two insulin monomer molecules with essentially similar conformation having quasi-twofold symmetry. Each molecule consists of two chains noted as A and B. The B chains' residues from 9 to 19 form a helix from which the initial and terminal residues turn into generally extended confor- 
mations. The A chain's residues are both compact and rest within the framework of the extended B chains and the B chain helices to which they are linked by disulphide bonds, A20-B19 and A7-B7. The interface of the two monomers is formed mostly between the two B chains.

A united-atom representation of insulin dimer was modeled using the GROMOS96 force field. The ionic strength was set to zero, so $\kappa=0$. To assess the effect of grid resolution on the calculation, the PBE was solved with the different meshes $\left(155^{3}, 105^{3}, 85^{3}, 65^{3}\right)$ within the FDSD simulation with the dielectric constant of the solute equal to 1 . The results show that the simulation quality does not sensitively depend on the grid resolution under certain resolution degree. This observation agrees with the earlier work ${ }^{17,29}$ that has demonstrated that in dynamics simulations, the use of a fine grid (around $0.5 \AA$ ) gives no obvious improvement over that using a coarse grid (about $1.0 \AA$ ). Use of a coarse grid (about $1 \AA$ ) leads to a significant reduction in time spent on the calculation of electrostatic interactions. For this reason, other FDSD simulations were performed with the $65^{3}$ mesh (about $1.0 \AA$ grid spacing). The GROMOS96 atomic charges and radii were used to represent the insulin dimer in the $\mathrm{PB}$ part of the calculation. The solute-solvent boundary was formed between the van der Waals envelope of the molecule and a probe solvent molecule with a sphere radius of $1.4 \AA$. The dielectric constant was 80 for the solvent. Boundary conditions were set to calculate the potential at each boundary point due to every charge in the system using the Debye-Hückel approximation (full Coulombic). The PB force was updated every time step. In order to establish a comparison and select a proper dielectric constant for the solute, a set of values $(1,2,4,6,8,10,13,15,17,20$, and 30$)$ were selected for each separate simulation.

For comparison, the MD simulation with explicit water and the conventional SD simulation in a vacuum were performed. A periodic box including 4,512 water molecules was used for the MD simulation. The short-range and long-range pair lists included atoms within 8 and $14 \AA$, respectively. In the conventional SD simulation, the friction coefficient was taken to be $91 \mathrm{ps}^{-1}$, weighted with the accessible area factor, and the relaxation time was set to $0.1 \mathrm{ps}$. In each simulation, the system was coupled to heat bath at $300 \mathrm{~K}$ with a coupling constant of $0.1 \mathrm{ps}$. The SHAKE algorithm ${ }^{30}$ was employed with a 2 -fs time step. After minimization, the FDSD simulations with a dielectric of 1 for the solute and with different grid resolutions were all performed for $20 \mathrm{ps}$, and any other simulation was performed for $500 \mathrm{ps}$. The trajectories were saved every 50 time steps.

\section{RESULTS AND DISCUSSION Effect on Dynamics of Grid Resolution}

In the $\mathrm{PB}$ calculation, the grid resolution is a main factor to decide the computer time. To get a balance between accuracy of calculation and computational power, an appropriate resolution should be selected, especially for dynamics simulation. Several 20-ps FDSD simulations with $155^{3}$ (about $0.3 \AA$ grid spacing), $105^{3}$ (about $0.5 \AA$ grid spacing), $85^{3}$ (about $0.7 \AA$ grid spacing), $65^{3}$ (about $1.0 \AA$ grid

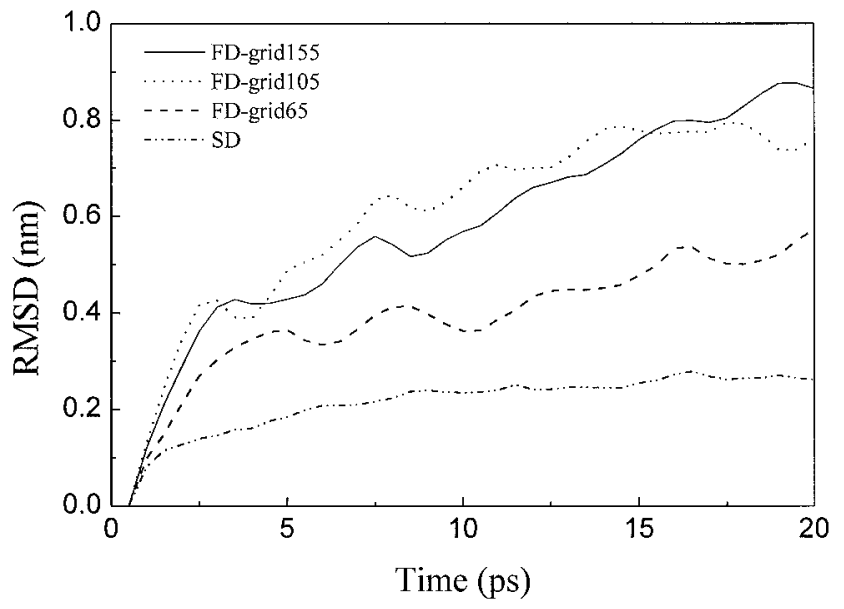

Fig. 1. Root mean square deviations of atomic positions vs. simulation time relative to the initial structure. Solid line: FDSD simulation with $155^{3}$ grid mesh. Dotted line: FDSD simulation with $105^{3}$ grid mesh. Dashed line: FDSD simulation $65^{3}$ grid mesh. Dash-dot-dotted line: SD simulation.

spacing) grid meshes were performed. For comparison with SD simulation, in which the dielectric value of the solute interior is set to1, the same constant is assigned for the solute in the FDSD simulations. The displacement of the generated structure from a reference structure as a function of simulation time (in terms of overall RMSD) gives a direct and obvious picture of changes in conformation during the simulation. Figure 1 shows the timedependence of the RMSD of atomic positions in the above FDSD simulations (skipped the case of $85^{3}$ grid mesh just for clarification) and SD simulation relative to the initial structure after minimization from the X-ray crystal structure. The RMSD obtained from SD simulation keeps a stable value of approximately $2.0 \AA$ after a short initial period, while the other RMSD values obtained in different FDSD simulations are increased with simulation time. At the time of $20 \mathrm{ps}$, the RMSD values approach a large value of more than $5 \AA$, especially for the FDSD simulations with a $0.3 \AA$ grid and a $0.5 \AA$ grid. This means that the protein is quickly denatured. Moreover, Figure 1 also indicates that the finer grid resolution ( $0.3 \AA$ in this case) does not improve the quality of dynamics simulation, at least under this protocol. The energies were also calculated for further comparison. Table I lists some types of energies calculated from the FDSD and SD simulations. For the calculation of electrostatic energy for the same initial conformation of insulin dimer, the three calculations with $155^{3}, 85^{3}, 65^{3}$ grid meshes give very closed values within an error no more than $4 \%$ between each other. And the calculations for the electrostatic solvation energy $\left(\mathrm{E}_{\mathrm{rf}}\right)$ for the initial conformation from the four PB solutions (especially for GRID155, GRID85, GRID65) are comparable (see Table I). This indicates that the results of the electrostatic energy calculation from above four grid resolutions are not far from each other in our method. As for the difference between the results obtained with FDSD and SD calculations, this is due to the different treatment on the electrostatics, especially for the electrostatic solvation effect. Table I also shows the positive reaction field energy $\left(\mathrm{E}_{\mathrm{rf}}\right)$ in 
TABLE I. Initial and Final Energies (Corresponding to the First and Second Data in Each Unit Box, Respectively) in Different Simulations of 20 ps Duration ${ }^{\dagger}$

\begin{tabular}{lcrrrrr}
\hline Simulation & $\mathrm{E}_{\mathrm{k}}$ & $\mathrm{E}_{\mathrm{el}}$ & $\mathrm{E}_{\mathrm{rf}}$ & $\mathrm{E}_{\mathrm{vdw}}$ & $\mathrm{P}_{\mathrm{a}}$ & $\mathrm{P}_{\mathrm{nonb}}$ \\
\hline \multirow{2}{*}{$\mathrm{SD}$} & 2,568 & $-4,414$ & 770 & $-3,374$ & $-6,427$ & $-7,788$ \\
& 2,576 & $-6,361$ & 968 & $-3,284$ & $-6,218$ & $-9,645$ \\
GRID155 & 2,579 & $-10,125$ & $-4,314$ & $-3,374$ & $-12,165$ & $-13,527$ \\
& 3,675 & $-10,525$ & $-6,868$ & $-1,687$ & $-8,095$ & $-12,213$ \\
GRID105 & 2,578 & $-9,685$ & $-4,825$ & $-3,374$ & $-10,539$ & $-13,045$ \\
& 3,058 & $-10,525$ & $-6,756$ & $-1,840$ & $-8,511$ & $-12,366$ \\
GRID85 & 2,575 & $-9,907$ & $-4,070$ & $-3,374$ & $-11,921$ & $-13,282$ \\
& 2,986 & $-10,264$ & $-6,168$ & $-2,103$ & $-8,719$ & $-12,367$ \\
GRID65 & 2,571 & $-10,252$ & $-4,015$ & $-3,390$ & $-12,288$ & $-13,649$ \\
& 2,906 & $-9,816$ & $-5,159$ & $-2,733$ & $-9,289$ & $-12,550$ \\
\hline
\end{tabular}

GRID155 denotes the FDSD simulation with $155^{3}$ grid mesh, and the GRID105, GRID85, GRID65 are alike. $E_{k}$ is the kinetic energy of the solute system, $E_{e l}$ the electrostatic energy (in which the self energy was subtracted), $\mathrm{E}_{\mathrm{rf}}$ the reaction field energy, $\mathrm{E}_{\mathrm{vdw}}$ the van der Waals interaction energy, $\mathrm{P}_{\mathrm{a}}$ the total potential energy. $P_{n o n b}$ the nonbonded interaction energy. All the energies are in unit of $\mathrm{kJ} / \mathrm{mol}$.

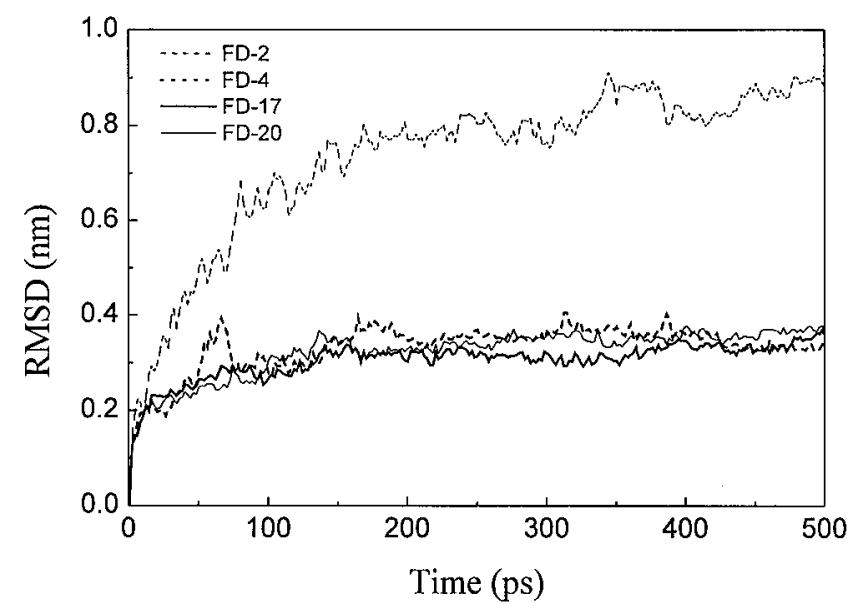

Fig. 2. Root mean square deviations of atomic positions vs. FDSD simulation time relative to the initial structure. Dashed line: FDSD simulation with $\epsilon=2$. Dotted line: FDSD simulation with $\epsilon=4$. Thick solid line: FDSD simulation with $\epsilon=17$. Thin solid line: FDSD simulation with $\epsilon=20$.

$\mathrm{SD}$ calculation, while the solvation energies $\left(\mathrm{E}_{\mathrm{rf}}\right)$ in the FDSD calculations are all negative. In fact, the SD simulation takes into poor account the solvation contribution to the energy. However, the variations of the van der Waals interactions $\left(\mathrm{E}_{\mathrm{vdw}}\right)$ in the four FDSD simulations are all much larger than that in the SD simulation. This implies that the relative positions between the solute atoms have a large displacement, and the structure of the solute may be changed. The variations of kinetic energies $\left(\mathrm{E}_{\mathrm{k}}\right)$ in the FDSD simulations are also large, which indicates that the dynamics are not stable. (Fig. 1; Table I)

Together with the above analysis for Figure 1 and Table I, an implication is obtained about the probably inappropriate value of the dielectric constant of the solute in the FDSD simulation, and the approximate Coulomb forces in the single $\mathrm{PB}$ calculation does not resulting in obvious affection. Consequently, a set of dielectric constants is tested, and a coarse grid of about $1 \AA$ spacing $\left(65^{3}\right.$ mesh $)$ is used in all the FDSD performances. The same coarse grid usage can also be found in David et al.'s work. ${ }^{17}$

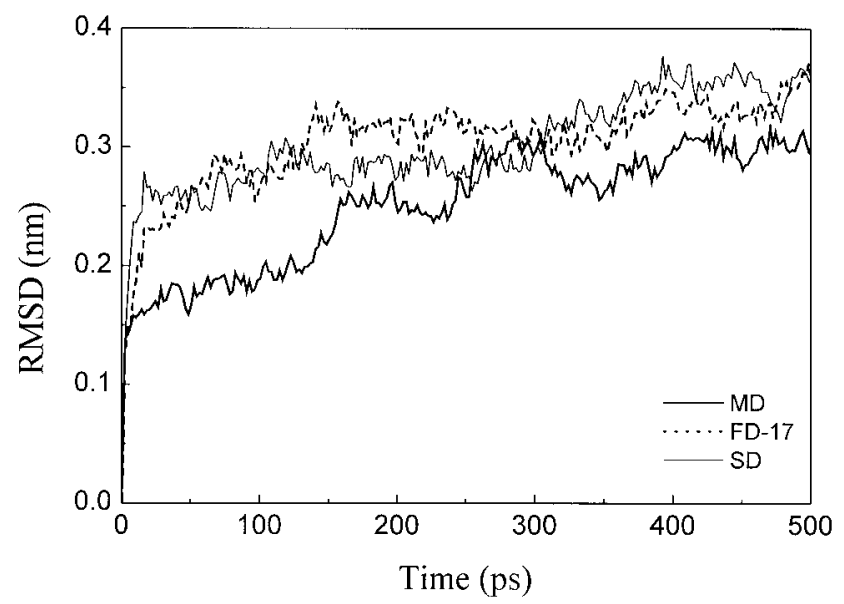

Fig. 3. Root mean square deviations of atomic positions vs. MD, FDSD, and SD simulation time relative to the initial structure. Thick solid line: MD simulation. Dotted line: FDSD simulation with $\epsilon=17$. Thin solid line: SD simulation.

\section{RMSD}

Figure 2 shows the time-dependence of the RMSD of atomic positions in the FDSD simulation relative to the initial structure after minimization from the X-ray crystal structure. For brevity, we use FD-n for denoting the FDSD simulation with a relative dielectric value of $n$. As shown in Figure 2, the RMSD obtained in a simulation with the dielectric constant equal to 2 (FD-2) reaches to a large value around $0.9 \mathrm{~nm}$. In fact, closer examination shows that the insulin dimer gradually assumes a state with two helices of each A chain unfolded as the simulation progresses. This indicates that a value of 2 is not a good choice for the dielectric constant in this model. When the dielectric constant is equal to or greater than 4, the RMSD obtained from different simulations is comparable, and much smaller. The FD-4 simulation produces an average RMSD value of $0.33 \mathrm{~nm}$, and the average RMSD value obtained from the FD-17 simulation decreases to $0.30 \mathrm{~nm}$, and that from the FD-20 simulation increases to $0.32 \mathrm{~nm}$ again. Figure 3 presents a comparison of the RMSD values 


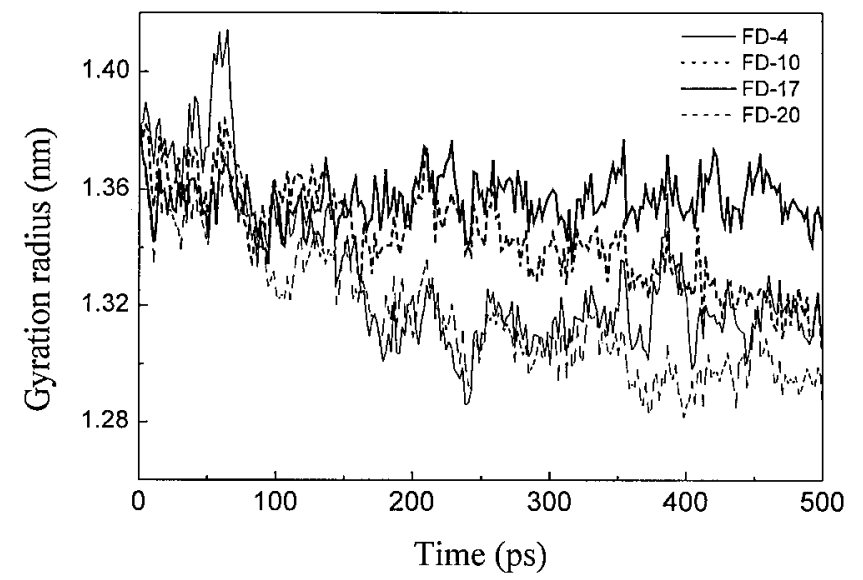

Fig. 4. Gyration radii as functions of FDSD simulation time. Thin solid line: FDSD simulation with $\epsilon=4$. Dotted line: FDSD simulation with $\epsilon=$ 10. Thick solid line: FDSD simulation with $\epsilon=17$. Dashed line: FDSD simulation with $\epsilon=20$.

as functions of simulation time for the MD, SD and FDSD simulations with the dielectric constant 17 . The maximum RMSD values in the series of the generated structures from initial structures in the MD, SD, and FD-17 simulations are $0.320,0.378$, and $0.370 \mathrm{~nm}$, respectively. Taking the system to be in a fully equilibrated state in the last 200 ps of the simulation time, the mean RMSD values averaged in this period of the MD, SD, and FDSD simulations are $0.291,0.344$, and $0.327 \mathrm{~nm}$, respectively. The above two sets of values indicate that the system in FDSD simulation with a dielectric value of 17 shows intermediate stability and flexibility relative to those in the full MD and conventional SD simulations. (Figs. 2, 3).

\section{Gyration Radius of Protein}

The gyration radius reflects molecular compactness shape. Figure 4 shows a different gyration radius in simulations with $\epsilon=4,10,17$, and 20 as a function of simulation time. It is interesting to find that in all FDSD simulations except for that with $\epsilon=17$, the gyration radius tends to decrease to a lower value in the later part of the simulation period. However, the gyration radius in simulation with $\epsilon=17$ keeps stable. The mean gyration radii in the eight simulations with $\epsilon=2,4,6,10,15,17,20$, and 30 are equal to, respectively, $1.330,1.330,1.337,1.344,1.343,1.357,1.318$, and $1.343 \mathrm{~nm}$. Apparently, in our FDSD simulations the conformation is changed more or less from its native state and the molecule becomes more compact than its initial structure, except in the simulation with $\epsilon=17$. The gyration radius obtained from the FDSD simulation with $\epsilon=17$ is compared with that in the MD and SD simulations in Figure 5. During most of the $500 \mathrm{ps}$ simulation time, the gyration radius in the FD-17 simulation lies between that in the MD and SD simulations, and this is also true of the mean gyration radius (1.363 nm for $\mathrm{MD}, 1.346 \mathrm{~nm}$ for $\mathrm{SD}$, and $1.357 \mathrm{~nm}$ for FD-17).

The system in the vacuum as shown in the SD simulation has the smallest value of the gyration radius. This result is in accord with the study by Wang et al. ${ }^{31}$ in which the gyration radius of a protein at a low hydration level is smaller than that at a high hydration level. This behavior

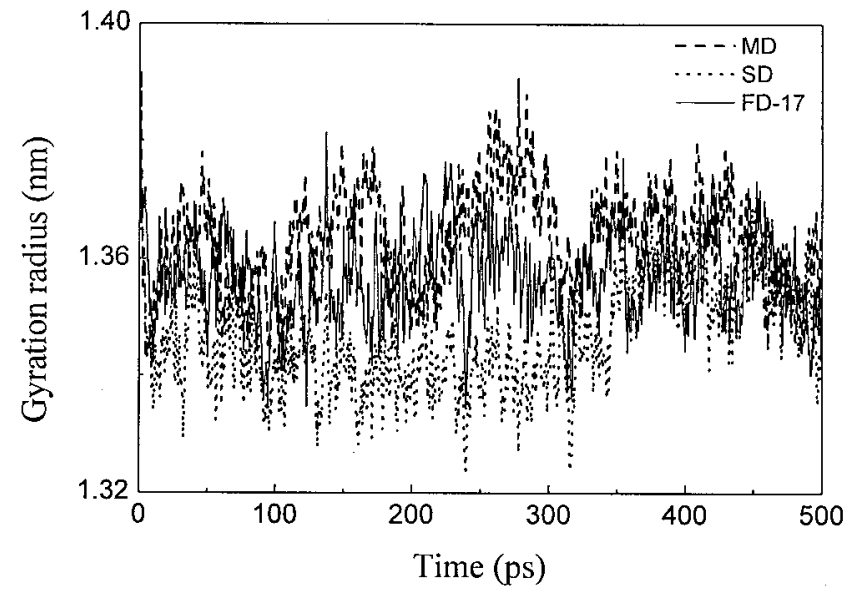

Fig. 5. Gyration radii as functions of MD, SD, and FDSD simulation time. Dashed line: MD simulation. Dotted line: SD simulation. Solid line: FDSD simulation with $\epsilon=17$.

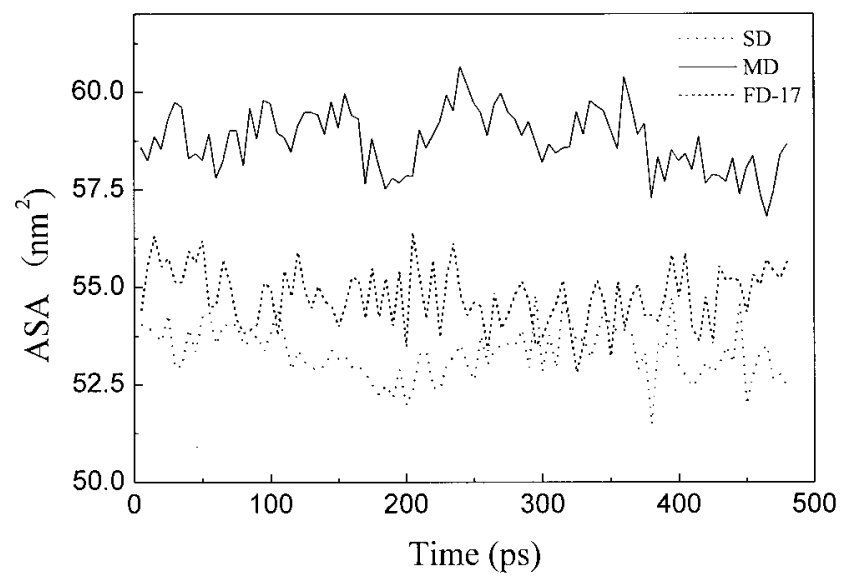

Fig. 6. The total ASA of the insulin dimer in MD, SD, and FD simulations. Solid line: MD simulation. Dotted line: SD simulation. Dashed line: FDSD simulation with $\epsilon=17$.

can be explained as the result of a tendency of both the intramolecular van der Waals and electrostatic interactions to minimize the surface of a protein at low hydration. Inclusion of solvent effect makes the gyration radius closer to that in the explicit solvent. In addition, Figure 5 shows that the molecular gyration radius keeps stable in three simulations, which is important and a necessary condition for simulations to generate an equilibrium state ensemble (Figs. 4, 5)

\section{Solvent ASA Analysis}

The solvent accessible surface area (ASA) is a direct measure for the interaction between solute and solvent, which in a simple way relates to the hydrophobic energy in the empirical calculation. ${ }^{5}$ Figure 6 gives a picture of the total ASA changes for the insulin dimer in the MD, SD, and FD-17 simulations. The ASA in the MD simulation has a maximal value, which reflects the strongest interaction between the solute and solvent in the explicit model, while the interaction decreases in the implicit treatment. However, the ASA keeps larger values in the FDSD 
TABLE II. Frequencies of Intramolecular Hydrogen Bonds Obtained From Different Simulation Techniques in Insulin Dimer (in Percentages)

\begin{tabular}{|c|c|c|c|c|c|c|}
\hline \multicolumn{2}{|l|}{ Donor } & \multicolumn{2}{|c|}{ Acceptor } & \multirow{2}{*}{$\begin{array}{c}\mathrm{MD} \\
44\end{array}$} & \multirow{2}{*}{$\begin{array}{c}\text { SD } \\
90\end{array}$} & \multirow{2}{*}{$\begin{array}{c}\text { FDSD } \\
17\end{array}$} \\
\hline 45Phe & $\mathrm{N}-\mathrm{H}$ & 98Tyr & $\mathrm{O}$ & & & \\
\hline 47Tyr & $\mathrm{N}-\mathrm{H}$ & 96Phe & $\mathrm{O}$ & 80 & 64 & 62 \\
\hline 96Phe & $\mathrm{N}-\mathrm{H}$ & 47Tyr & $\mathrm{O}$ & 71 & 91 & 55 \\
\hline 98Tyr & $\mathrm{N}-\mathrm{H}$ & 45Phe & $\mathrm{O}$ & 85 & 88 & 46 \\
\hline 27Leu & $\mathrm{N}-\mathrm{H}$ & 6Cys & $\mathrm{O}$ & 38 & 94 & 16 \\
\hline 7Сys & $\mathrm{N}-\mathrm{H}$ & $3 \mathrm{Val}$ & $\mathrm{O}$ & 89 & 82 & 62 \\
\hline 43Arg & $\mathrm{N}-\mathrm{H}$ & 40Cys & $\mathrm{O}$ & 48 & 26 & 18 \\
\hline 44Gly & $\mathrm{N}-\mathrm{H}$ & 41Gly & $\mathrm{O}$ & 60 & 0 & 0 \\
\hline 44Gly & $\mathrm{N}-\mathrm{H}$ & 21Asn & $\mathrm{O} 1$ & 0 & 99 & 0 \\
\hline 1Gly & $\mathrm{N}-\mathrm{H} 2$ & 51Ala & $\mathrm{O} 1$ & 0 & 87 & 0 \\
\hline 52Gly & $\mathrm{N}-\mathrm{H} 2$ & 102Ala & $\mathrm{O} 2$ & 0 & 60 & 3 \\
\hline
\end{tabular}

simulation than that in the SD simulation. This shows that the improvement in taking into account the solvation effect has been achieved in the FDSD simulation. Due to polarization included in the $\mathrm{PB}$ solution, the polar residues tend to be exposed outside in the FDSD simulation more than in the SD simulation. The data of the ASA of polar residues has the same pattern, which also supports this argument (not shown here) (Fig. 6).

\section{Hydrogen Bonding Analysis}

The structure of intramolecular H-bonds is an excellent measure of structural integrity. Table II reports the results of H-bond analysis of insulin dimer obtained from trajectories obtained with MD, SD, and FDSD with $\epsilon=17$. The criteria used to determine an H-bond are purely geometric. For each coordinate set, every potential donoracceptor pair is tested and considered to form an H-bond if the distance between hydrogen and acceptor is less than $0.25 \mathrm{~nm}$ and the donor-hydrogen-acceptor angle is larger than $135^{\circ}$. The H-bond frequencies are obtained from the registered occurrences of the simulation trajectory frames.

Table II lists several H-bond donor and acceptor pairs located in different part of the insulin dimer and their occupancies. In the region B24 (45Phe) to B26 (47Tyr), the chain is involved in antiparallel $\mathrm{H}$-bonding with its symmetry-related equivalents (96Phe to 98Tyr). Except for the donor and acceptor pair between 47Tyr $(\mathrm{N}-\mathrm{H})$ and $96 \mathrm{Phe}$ $(\mathrm{O})$, the frequencies of the other three interfacial antiparallel H-bonds among residues 45, 47 and 96, 98 are lower in the MD simulation than in the SD simulation. These frequencies are still lower in the FDSD simulation, but not far from the results obtained with the MD simulation. This phenomenon may be due to the effects of screening and polarization in the MD and FDSD simulations. Especially, the FDSD simulation with $\epsilon=17$ may take into account an "excess" solvent effect (imposing strong screening effect on the solute), which results in weaker electrostatic interactions and then unfavorable formation of H-bond. And another possible explanation for the even lower H-bonds occurrences is the approximate finite-difference Coulomb forces and probably also the spurious forces arising from the position-dependence of the self-energy terms of the gridded charges. This noise may be partially responsible for the low occurrences of H-bonds in the FDSD run. The same case occurs for the H-bond network between the A and $\mathrm{B}$ chains in a monomer such as that between 27Leu $(\mathrm{N}-\mathrm{H})$ and $6 \mathrm{Cys}(\mathrm{O})$, the helical contact between 7Cys $(\mathrm{N}-\mathrm{H})$ and $3 \mathrm{Val}(\mathrm{O})$, and the contact at the bend between $43 \mathrm{Arg}(\mathrm{N}-\mathrm{H})$ and $40 \mathrm{Cys}(\mathrm{O})$. However, the structure between 44Gly-NH and 41Gly-O that is prevalent during the MD simulation, breaks down in both the SD and the FDSD simulations. The final three H-bond pairs in Table II show distinct behavior in the three different simulations. The frequencies of these three H-bonds are zero or nearly zero in the MD and FDSD simulations, whereas the SD simulation generates high occupancies of the H-bonds. The residues in these three main chain $\mathrm{H}$-bond contacts are near the molecular surface and are solvated in aqueous solution, and in the MD simulation the explicit water molecules compete to form $\mathrm{H}$-bond with the solute as donors or acceptors. The implicit solvation forces introduced in the FDSD simulation succeed in reducing H-bond interactions among surface residues.

\section{Molecular Structure}

Most of the above structural analyses support the validity of the FDSD simulation with dielectric of 17 for solute interior. However, the question is that did some low occurrences of $\mathrm{H}$-bonds imply that the inner structure of the insulin dimer has been distorted in the FDSD simulation with an interior dielectric constant of 17? Three snapshots got at the end of the 500-ps MD, SD, and FD-17 simulations, respectively, are shown in Figure 7. Compared with the structure obtained from MD simulation, the structures are kept very well in the SD and FDSD $(\epsilon=$ 17) simulations. The RMSD calculated from the superposition of the MD and SD structures is $0.259 \mathrm{~nm}$, and the RMSD for the two MD and FDSD structures is $0.264 \mathrm{~nm}$. The six helices and two sheets in each conformation are stable during the three simulations. As shown in Figure 7, only some small parts, i.e., a strand at the end of B chain between residues 22Phe and Asn24, are flexible with large magnitude. Even for the two long extended parallel coils of B chain (from 44Gly to Lys50) on the interface of the dimer, the closeness is tightly kept. Figure 7 indicates that the FDSD $(\epsilon=17)$ run does not break the inner structure of the dimer. The noise of the force in the present method can only, if it does, result in a relative small positional change and does not impose much influence on the dynamic and structural properties of protein (Fig. 7).

\section{Energy Analysis}

The energy conservation and its fluctuation are of interest in the simulated equilibrium state. Figure 8 shows the fluctuating temperature of the solute system with respect to simulation time in the MD, SD, and FDSD $(\epsilon=17)$ simulations. In fact, the temperature reflects the kinetic energy of the system. As shown in Figure 8, the temperature fluctuation in the FDSD and MD simulations are smaller and more stable than in the SD simulation, which is due to the solvation effect. In the vacuum, as in the SD simulation, the atoms of solute vibrate more violent than that in solvent (Fig. 8). 

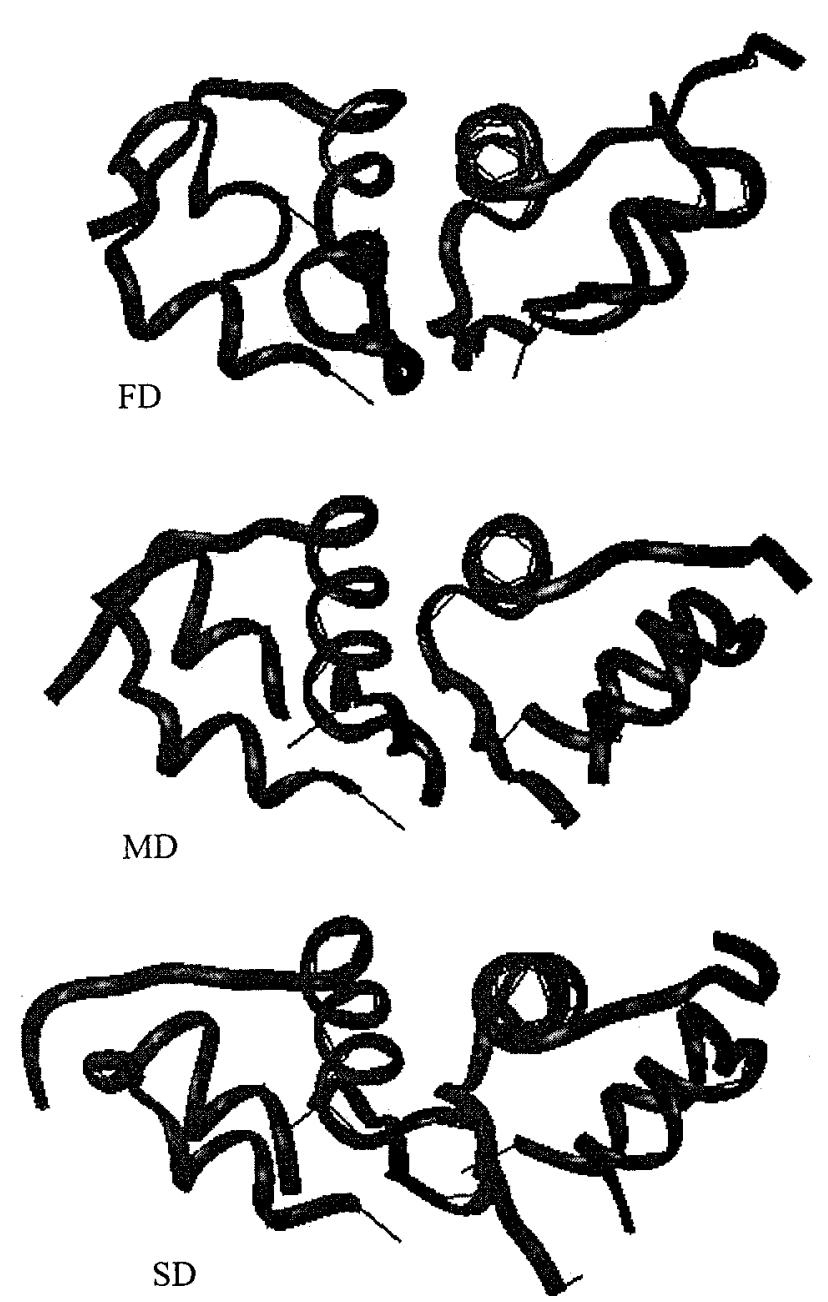

Fig. 7. The structure snapshots got at the end of the three different 500-ps simulations: MD, SD simulations, and the FDSD simulation with $\epsilon=17$.

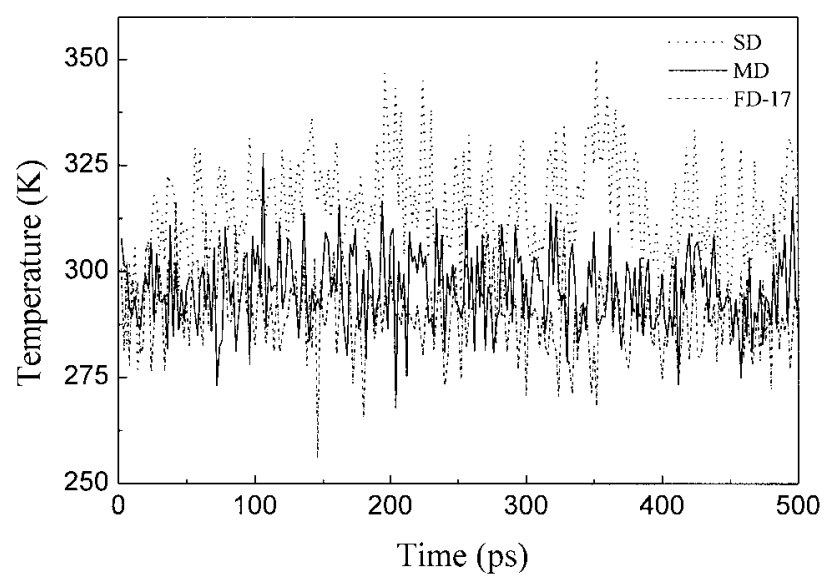

Fig. 8. The temperature fluctuation of the solute system with respect to simulation time in MD, SD, and FDSD $(\epsilon=17)$ simulations. Solid line: MD simulation. Dotted line: SD simulation. Dash-dotted line: FDSD simulation with $\epsilon=17$.

Figure 9 presents the reaction field energy (E-RF) (electrostatic solvation energy), electrostatic energy (EELE) and the total potential (E-POT) of insulin dimer as a

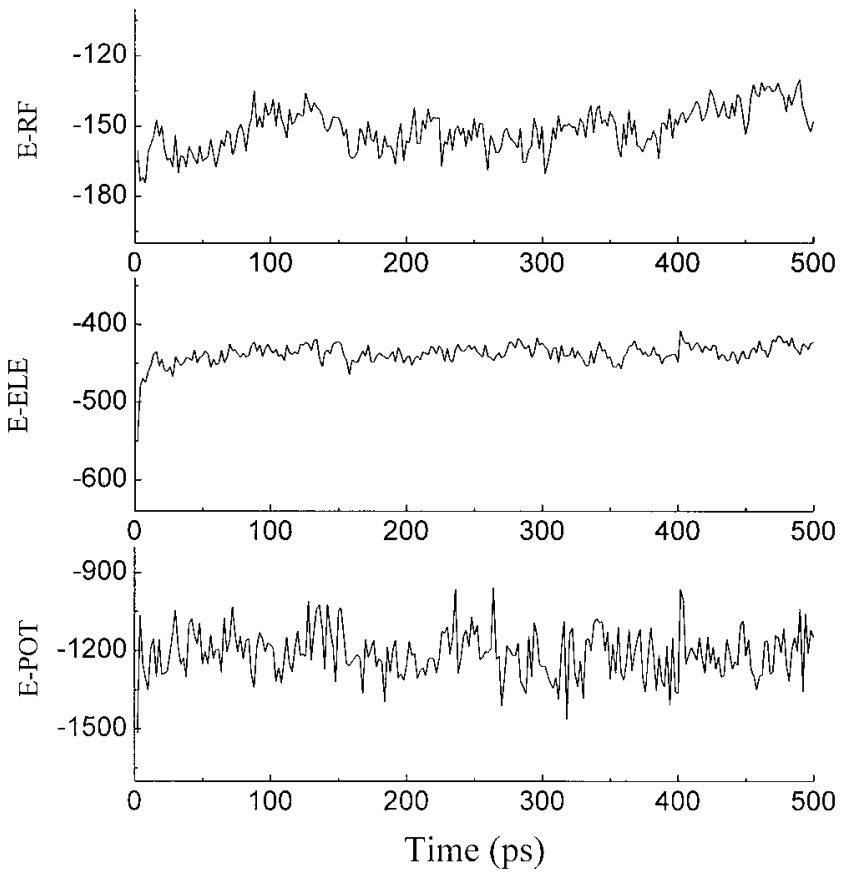

Fig. 9. The energetic variations with simulation time in FDSD simulation with $\epsilon=17$. E-RF denotes the reaction field energy. E-ELE is the electrostatic energy. E-POT is the total potential. All the energies are in unit of $\mathrm{kJ} / \mathrm{mol}$.

function of simulation time for the FDSD simulation with $\epsilon=17$. The energies keep stable well. The average E-RF and its error in the 500-ps duration is $-151 \pm 9 \mathrm{~kJ} / \mathrm{mol}$, the average E-ELE and its error $-437 \pm 12 \mathrm{~kJ} / \mathrm{mol}$, and the average E-POT and its error $-1,210 \pm 91 \mathrm{~kJ} / \mathrm{mol}$. However, the absolute values of the energies shown in Figure 9 cannot be comparable with that calculated in the MD, SD, or even the FDSD with $\epsilon=1$ simulations as listed in Table I. Since the dielectric constant 17 , as a factor in the electrostatic interaction, results in a decrease in the electrostatic energies, this figure only gives a view of energy conservation with simulation time (Fig. 9).

\section{CONCLUSIONS}

The procedure incorporating the electrostatic force entirely determined by the FDPB method into the SD simulation has demonstrated its reliability in the protein modeling. The GROMOS96 force field is used in all simulations and is adaptable to the FDPB method. The analysis results on structural, dynamics, and energetics properties of the insulin dimer obtained with different FDSD simulations show that the coarse grid does not reduce the calculation accuracy in the dynamics simulation in this method and a single PB calculation can also lead to the good quality of a dynamics simulation. The problem still remains if the noise of force by a single $\mathrm{PB}$ calculation would be averaged more or less in the dynamics simulation. The results obtained with FDSD simulation with relative dielectric constant of 17 assigned to the solute interior are in similar accuracy compared to the explicitsolvent MD simulation. This indicates that solvent effects on structural and dynamic properties can be reflected in 
the FDSD simulation, which is due to that the dielectric constant accounts for the polarization and screening effect of solvent. Also, this work indicates that the dielectric constant is a key parameter in a simulation procedure in which the FDPB method is used to treat the full electrostatics. Although the artificial interior dielectric constant 17 seems rather high, it appears an appropriate choice to use in the present context. Moreover, it is close to the selection of 20 used in Schaefer et al. ${ }^{15}$ and Antosiewicz et al. ${ }^{16}$ in which the FDPB method is used to calculate the $\mathrm{pK}_{\mathrm{a}}$ shift. We expect the same appropriateness of this value in the MD simulations of the different systems with the similar size.

For a more detailed simulation, the incorporation of the force arising from non-polar solvation effect into dynamics simulation is the further extension of this work as well as the more accurate calculation of the PB electrostatic force.

The primary advantage of using the implicit model is the time savings afforded by replacing explicit water molecules with the continuum. Note, however, that the time saved is strongly dependent on the grid size and the updating frequency of the PB force. Setting the CPU time per dynamics picosecond for the MD simulation with explicit water molecules as an arbitrary reference of 100 , we find that in our FDSD approach, the CPU time consumed per picosecond is 25 when the calculation of the $\mathrm{PB}$ force is performed every 3 time steps, and 60 when the PB forces are updated at each time step, while in the conventional SD simulation it is only 8 . Advances in numerical methods for solving the FDPB will make FDSD approach more attractive.

\section{ACKNOWLEDGMENTS}

We thank Professor J. Hermans for comments on this work. We also thank Professor W.F. van Gunsteren for kindly providing us with the GROMOS96 package.

\section{REFERENCES}

1. Brooks C, Karplus M, Pettitt M. Proteins: A theoretical perspective of dynamics, structure and thermodynamics. Adv Chem Phys 1987;71:1-259.

2. Li A, Daggett V. Identification and characterization of the unfolding transition state of chymtrypsin inhibitor 2 by molecular dynamics simulations. J Mol Biol 1996;257:412-429.

3. Bonvin AMJJ, Van Gunsteren WF. $\beta$-Hairpin stability and folding: molecular dynamics studies of the first $\beta$-hairpin of tendamistat. J Mol Biol 2000;296:255-268.

4. Sharp KA, Nicholls A, Fine RF, Honig B, Reconciling the magnitude of the microscopic and macroscopic hydrophobic effects. Science 1991;252:106-109.

5. Eisenberg D, Mclachan AD, Solvation energy in protein folding and binding. Nature 1986;319:199-203.

6. Zauhar RJ. The incorporation of hydration forces determined by continuum electrostatics into molecular mechanics simulations. J Comp Chem 1991;12:575-583.

7. Sharp K. Incorporating solvent and ion screening into molecular dynamics using the finite-difference Poisson-Boltzmann model. J Comp Chem 1991;12:454-468.

8. Gilson MK, Davis ME, Luty BA, McCammon JA. Computation of electrostatic forces on solvated molecules using the PoissonBoltzmann equation. J Phys Chem 1993;97:3591-3600.
9. Gilson MK, McCammon JA, Madura JD. Molecular dynamics simulation with a continuum electrostatic model of the solvent. J Comput Chem 1995;16:1081-1095.

10. Wang CX, Wan SZ, Xiang ZX, Shi YY. Incorporating hydration force determined by boundary element method into stochastic dynamics. J Phys Chem 1997;102:230-235.

11. Lu BZ, Wang CX, Chen WZ, Wan SZ, Shi YY. A stochastic dynamics simulation study associated with hydration force and friction memory effect. J Phys Chem B 2000;104:6877-6883.

12. Marrone TJ, Gilson MK, McCammon JA, Comparison of continuum and explicit models of solvation: potentials of mean force for alanine dipeptide. J Phys Chem 1996;100:1439-1441.

13. Honig B, Nicholls A. Classical electrostatics in biology and chemistry. Science 1995;268:1144-1149.

14. You TJ, Bashford D. Conformation and hydrogen ion titration of proteins: a continuum electrostatic model with conformational flexibility. Biophys J 1995;69:1721-1733.

15. Schaefer M, Sommer M, Karplus M. pH-dependence of protein stability: absolute electrostatic free energy differences between conformations. J Phys Chem B 1997;101:1663-1683.

16. Antosiewicz J, McCammon JA, Gilson MK. Prediction of pHdependent properties of proteins. J Mol Biol 1994;238;415-436.

17. David L, Luo R, Gilson MK. Comparison of generalized Born and Poisson methods: energetics and dynamics of HIV protease. J Comp Chem 2000;21:295-309.

18. Calimet N, Schaefer M, Simonson T. Protein molecular dynamics with the generalized Born/ACE solvent model. Proteins 2001;45: 144-158.

19. Gilson MK, Honig B. The inclusion of electrostatic hydration energies in molecular mechanics calculations. J Comp Aided Mol Des 1991;5:5-20.

20. Elcock AH, McCammon JA. The low dielectric interior of proteins is sufficient to cause major structural changes in DNA on association. J Amer Chem Soc. 1996;118:3787-3788.

21. Elcock AH, Potter MJ, McCammon JA. Application of PoissonBoltzmann solvation forces to macromolecular simulations. In: van Gunsteren WF, Wilkinson AJ, Weiner PK, editors. Computer simulation of biomolecular system, Vol. 3. Dordrecht: Kluwer Academic Publishers; 1997. p 244-261.

22. Cheng W, Wang CX, Chen WZ, Xu YW, Shi YY, Investigating the dielectric effects of channel pore water on the electrostatic barriers of the permeation ion by the finite difference PoissonBoltzmann method. Eur Biophys J 1998;27:105-112.

23. van Gunsteren WF, Billeter SR, Eising AA, Hünenberger PH, Krüger P, Mark AE, Scott WRP, Tironi IG. Biomolecular simulation: the GROMOS96 manual and user guide. Zürich, Switzerland: vdf Hochschuverlag; 1996.

24. Schutz CN, Warshel A. What are the dielectric "constants" of proteins and how to validate electrostatic model?. Proteins 2001; 44:400-417

25. Nicolls A, Honig B. A rapid finite difference algorithm, utilizing successive over-relaxation to solve the Poisson-Boltzmann equation. J Comp Chem 1991;12:435-445.

26. van Gunsteren WF, Berendsen HJC, A leap-frog algorithm for stochastic dynamics. Mol Simul 1988;1:173-185.

27. Madura JD, Davis ME, Gilson MK, Wade RC, Luty BA, McCammon JA. Biological applications of electrostatic calculations and Brownian dynamics simulations. Rev Comp Chem 1994;5:229-267.

28. Baker EN, Blundell TL, Cutfield JF, Cutfield SM, Dodson EJ, Dodson GG, Crowfoot Hodgkin DM, Hubbard RE, Isaacs NW, Reynolds CD, Sakabe K, Sakabe N, Vijayan NM. The structure of 2Zn pig insulin crystals at 1.5 angstroms resolution. Phil Trans R Soc Lond B 1988;319:369-456.

29. Smart JL, Marrone TJ, McCammon JA. Conformational sampling with Poisson-Boltzmann forces and a stochastic dynamics/Monte Carlo method: application to alanine dipeptide. J Comp Chem 1997;14:1750-1759.

30. Ryckaert JP, Ciccotti G, Berendsen HJC, Numerical integration of the cartesian equations of motion for a system with constraints: molecular dynamics of n-alkanes. J Comp Phys 1977;23:327-341.

31. Wang CX, Bizzarri AR, Xu YW, Cannistraro S, MD of plastocyanin: structure and dynamics. Chem Phys 1994;183:155-166. 